\title{
La rougeole en France
}

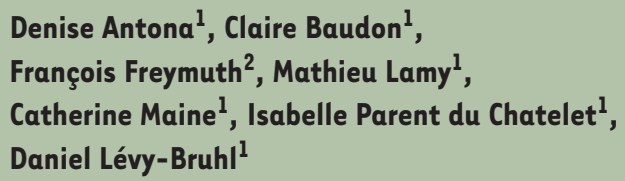

> Entre janvier 2008 et mai 2012, plus de 22000 cas de rougeole ont été déclarés en France. L'incidence la plus élevée a été observée chez les enfants de moins de un an, et plus de $50 \%$ des cas ont été déclarés chez de jeunes adultes. Près de 5000 patients ont été hospitalisés, dont 1023 atteints de pneumonie, 27 d'encéphalite/ myélite; 10 patients sont décédés. Cette situation résulte d'une couverture vaccinale insuffisante, hétérogène, avec constitution progressive d'un réservoir de sujets réceptifs permettant la circulation du virus. Si la couverture avec deux doses de vaccin a augmenté pour les enfants, améliorer celle des jeunes adultes demeure un objectif majeur pour éliminer la maladie et, en particulier, pour pouvoir protéger les populations vulnérables ne pouvant être vaccinées (enfants de moins d'un an, patients immunodéprimés, femmes enceintes). <

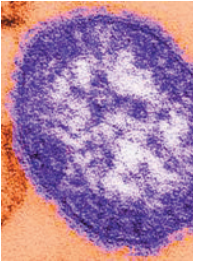

\author{
${ }^{1}$ Institut de veille sanitaire, \\ 12, rue du Val d'Osne, \\ 94415 St Maurice Cedex, \\ France ; \\ ${ }^{2}$ Centre national de référence \\ virus de la rougeole \\ et paramyxoviridae, \\ Laboratoire de virologie, \\ Centre hospitalo- \\ universitaire (CHU) de Caen, \\ avenue Georges Clémenceau, \\ 14033 Caen Cedex 9, France. \\ d.antona@invs.sante.fr
}

2015 de l'objectif d'élimination de la rougeole. Quatre domaines d'action clés ont été identifiés pour atteindre cet objectif :

- obtenir et maintenir un niveau de couverture vaccinale de $95 \%$ avec injection de deux doses en routine;

- assurer des actions de vaccination complémentaires ciblant les populations réceptives ;

- renforcer la surveillance en incluant l'investigation autour des cas et leur confirmation biologique ;

délivrer une information de qualité, accessible aux professionnels de santé ainsi qu'au grand public.

\section{Rappel clinique}

La rougeole est due à un virus à ARN de la famille des Paramyxoviridae, du genre Morbillivirus. La transmission est aérienne, de personne à personne, mais peut aussi se faire via du matériel récemment souillé par des sécrétions nasopharyngées. À la suite d'un contage, l'incubation dure de 10 à 12 jours. La phase prodromique (deux à quatre jours) associe malaise général, fièvre, catarrhe avec coryza, conjonctivite et/ou trachéobronchite. La température devient ensuite très élevée au cours des quatre jours suivants, pouvant atteindre $40^{\circ} \mathrm{C}$. Un énanthème buccal

Vignette : virus de la rougeole - (c) Cynthia Goldsmith/CDC. 


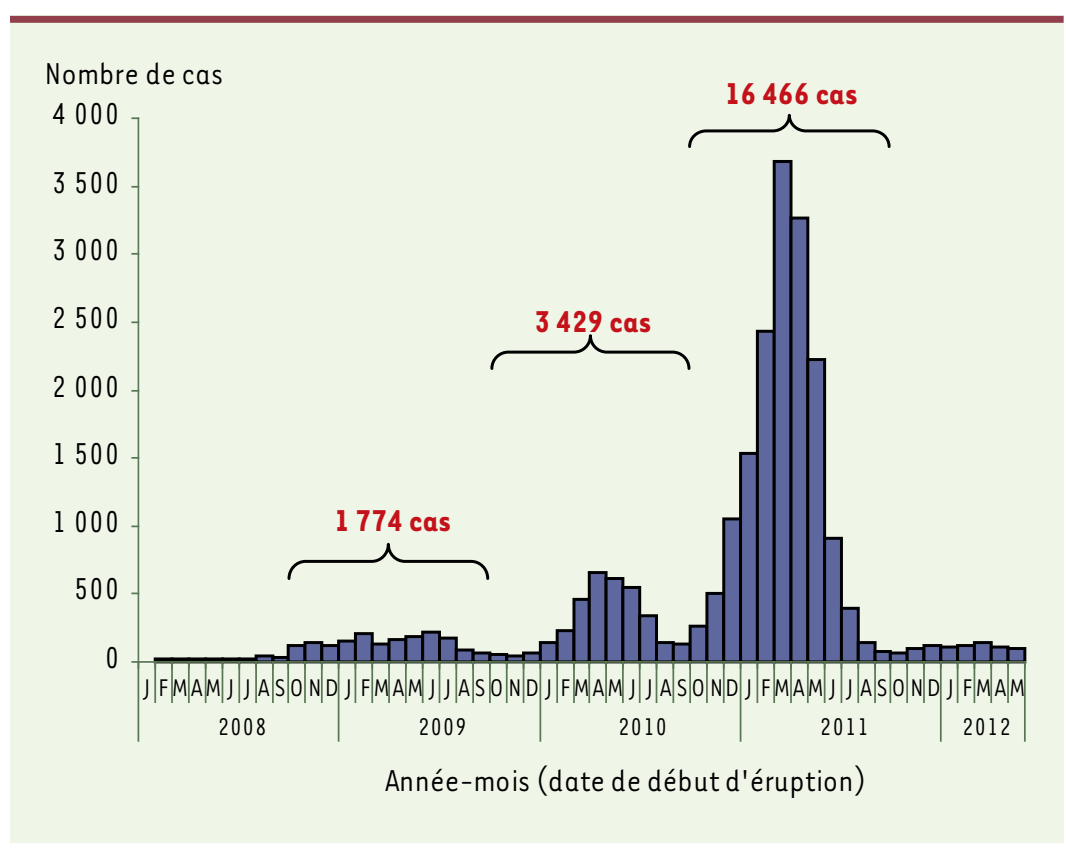

Figure 1. Nombre de cas de rougeole déclarés par mois. Données de déclaration obligatoire, France, janvier 2008-mai 2012 (source : chiffres de déclarations obligatoires, InVS).

grossesse peut être à l'origine d'une rougeole congénitale avec, pour le nouveau-né, non seulement un risque de pneumopathie et de décès, mais aussi un risque majoré de panencéphalite sclérosante subaiguë de survenue précoce.

La létalité est de 0,5 à 1 cas pour mille dans les pays industrialisés, mais peut être très élevée dans les pays en développement : estimée en moyenne à $7,4 \%$, elle peut atteindre jusqu'à $32 \%$ par exemple dans les conditions qui prévalent dans les camps de refugiés $[1,2]$.

\section{Contexte épidémiologique français}

apparaît et le signe de Koplick ${ }^{1}$ peut alors être mis en évidence. L'éruption apparaît en moyenne 14 jours après le contage, caractérisée par un érythème maculopapuleux, sans intervalle de peau saine et disparaissant à la vitropression. L'éruption débute au niveau de la face, derrière les oreilles et le cou, gagnant ensuite progressivement le tronc et les membres en trois à quatre jours. Elle disparaît ensuite en trois à quatre jours, en suivant le même ordre que lors de son apparition. Les patients sont contagieux jusqu'à cinq jours avant et après le début de l'éruption, avec un maximum de contagiosité lors de la phase prodromique de la maladie. L'infection confère une immunité à vie, y compris chez les personnes pauci symptomatiques.

Dans les pays industrialisés, les principales complications décrites sont les suivantes: otite moyenne aiguë (7 à $9 \%$ des cas), diarrhée ( $8 \%$ ), pneumonie ( 1 à $6 \%$ ), encéphalite ( 0,5 à 1 pour mille cas), et une complication tardive rare, la panencéphalite sclérosante subaiguë dans 1 cas pour 100000 rougeoles : il s'agit d'une dégénérescence du système nerveux central toujours mortelle, liée à la persistance du virus à ce niveau.

Le risque de complications est plus important chez les enfants âgés de moins d'un an ainsi que chez les adultes, en particulier chez les personnes immunodéprimées, mais aussi chez les femmes enceintes, pour elles-mêmes comme pour leur enfant. Le risque majeur pour la mère est celui d'une pneumopathie avec syndrome de détresse respiratoire aiguë pouvant aller jusqu'au décès. Le virus de la rougeole passe la barrière placentaire, et s'il n'est pas à l'origine de malformations fœtales, il peut en revanche entraîner une fausse couche ou un accouchement prématuré. De plus, une contamination en fin de

${ }^{1}$ Signe de Koplick : semis de petites taches blanchâtres sur fond érythémateux sur la muqueuse jugale, en face des prémolaires. II apparaît lors de la phase d'invasion dès la $36^{\mathrm{e}} \mathrm{h}$ et persiste jusqu'à l'éruption. II est considéré comme pathognomonique de la rougeole.
Pour atteindre en France l'objectif d'élimination de la rougeole fixé par l'OMS-Europe, un plan national a été mis en place en 2005 [3]. La rougeole est redevenue une maladie à déclaration obligatoire (DO) en juillet 2005 , avec confirmation biologique des cas. Un appui à la confirmation biologique et à la caractérisation des virus, notamment à partir de prélèvements salivaires, a été mis en place avec le centre national de référence ${ }^{2}$ (CNR). En termes de prévention, depuis 1983 le calendrier vaccinal français inclut la vaccination $d u$ nourrisson contre la rougeole, avec une $2^{\mathrm{e}}$ dose recommandée depuis 1996. Actuellement, la $1^{\text {re }}$ dose de vaccin rougeole-rubéole-oreillons (RRO) est recommandée à l'âge de 12 mois et la $2^{\mathrm{e}}$ dose entre 13 et 24 mois. Des recommandations de rattrapage vaccinal ciblent non seulement les enfants et adolescents, mais également les jeunes adultes et les professionnels de santé. Ainsi toute personne née depuis 1980 devrait avoir reçu au total deux doses de vaccin RRO, en respectant un délai minimum d'un mois entre les deux doses, quels que soient les antécédents vis-à-vis des trois maladies [4]. Des mesures préventives spécifiques visant à stopper la diffusion du virus dès la survenue d'un cas ou en situation de cas groupés ont également été préconisées [5]. Alors qu'en 2006 et 2007, avec moins de 50 cas annuels déclarés, l'incidence des cas déclarés était inférieure au seuil de 0,1/100 000 habitants défini par l'OMS pour l'élimination de la maladie, une résurgence de la rougeole en France a été détectée début 2008 et

\footnotetext{
${ }^{2}$ http://www.chu-caen.fr/service-129.html
} 


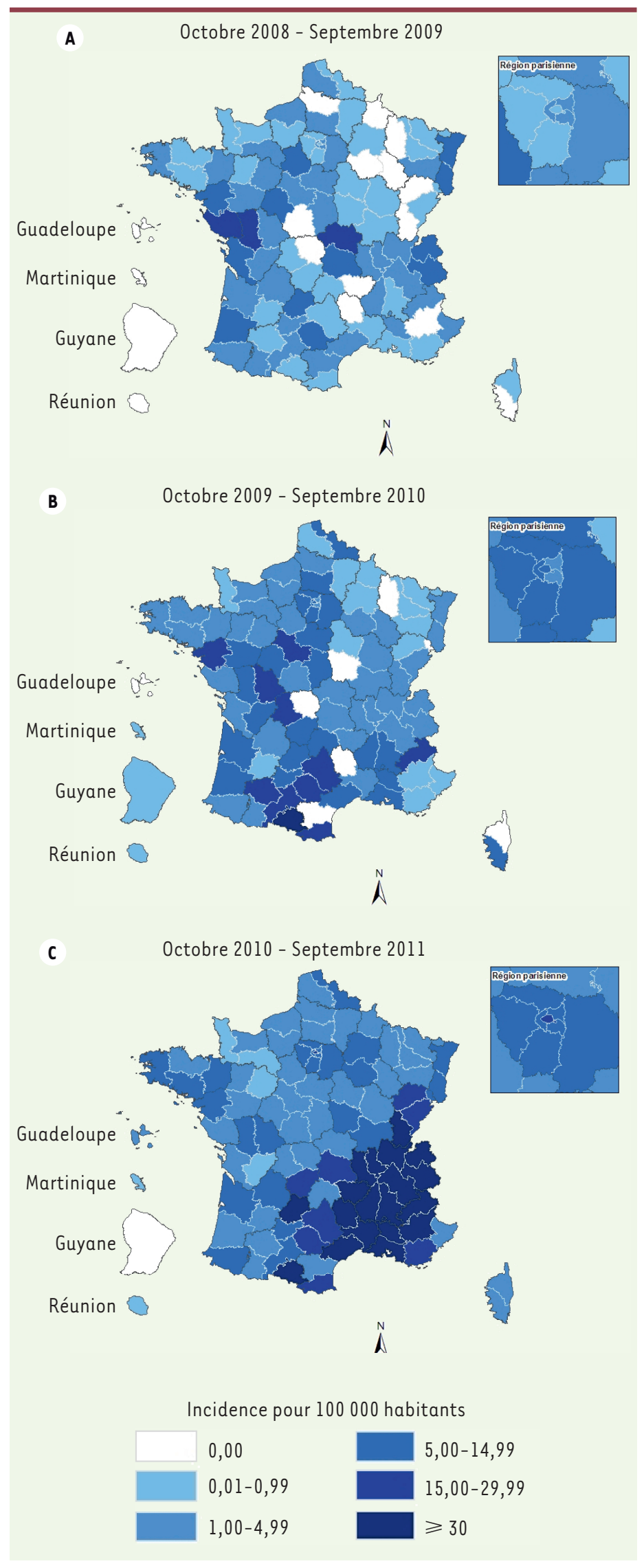

Figure 2. Distribution géographique des cas de rougeole déclarés. Évolution au cours des trois vagues épidémiques ( $A, B$, C), France, octobre 2008-septembre 2011 (source : chiffres de déclarations obligatoires, InVS).

s'est considérablement intensifiée ensuite, avec près de 22800 cas déclarés de janvier 2008 à mai 2012. La distribution mensuelle des cas a montré une augmentation notable du nombre des déclarations de rougeole dès l'automne 2008 avec trois vagues épidémiques (Figure 1). Un total de 21669 cas a été déclaré entre octobre 2008 et septembre 2011, et l'augmentation du nombre des cas approche un facteur dix entre la $1^{\text {re }}$ vague d'octobre 2008 à septembre 2009 (1774 cas) et la $3^{e}$ vague d'octobre 2010 à septembre 2011 (16 466 cas). L'incidence annuelle est ainsi passée de 2,7 cas pour 100000 habitants durant la $1^{\text {re }}$ vague à 5,2 durant la $2^{\mathrm{e}}$, pour atteindre 25,6 cas pour 100000 habitants durant la $3^{e}$ vague. L'ensemble du territoire national a été touché. Au cours de la $1^{\text {re }}$ vague, l'incidence la plus élevée était observée en Auvergne avec 12,8 cas pour 100000 habitants, suivie lors de la $2^{\mathrm{e}}$ vague par les régions Midi-Pyrénées et Limousin avec respectivement 15,7 et 13,0 cas pour 100000 habitants. Mais au cours de la $3^{e}$ vague, la circulation du virus s'est intensifiée de façon majeure dans le quart Sud- $\varepsilon$ st de la France. Les taux d'incidence en Rhône-Alpes, Provence-Alpes-Côte d'Azur et Languedoc-Roussillon ont alors atteint respectivement $97,2,53,9$ et 48,3 cas pour 100000 habitants (Figure 2).

L'analyse des cas a souligné le risque de contracter la maladie dans des tranches d'âges où la maladie est plus sévère puisque plus de la moitié des patients avaient plus de 15 ans et que, parmi ceux-ci, un sur trois était hospitalisé. L'âge médian des patients déclarés a augmenté au cours des trois vagues, de 12 ans pour la $1^{\text {re }}$ à 16 ans pour la dernière. On note aussi, de façon également préoccupante, une incidence très élevée chez les nourrissons de moins d'un an (Figure 3), qui ne sont pas protégés par des anticorps maternels et trop jeunes pour être vaccinés, avec en particulier 13 cas d'infection néonatale. Le bilan de l'épidémie est lourd puisqu'elle a entraîné, entre janvier 2008 et mai 2012, I'hospitalisation de 4980 patients dont 1023 étaient atteints de pneumopathie virale ou bactérienne grave, 27 de complication neurologique à type d'encéphalite ou de myélite, et on a dénombré 10 décès. La majorité de ces décès est survenue chez des personnes jeunes (neuf avaient moins de 30 ans). Sept d'entre elles présentaient un déficit immunitaire, et n'avaient donc pas pu être protégées par la vaccination. 


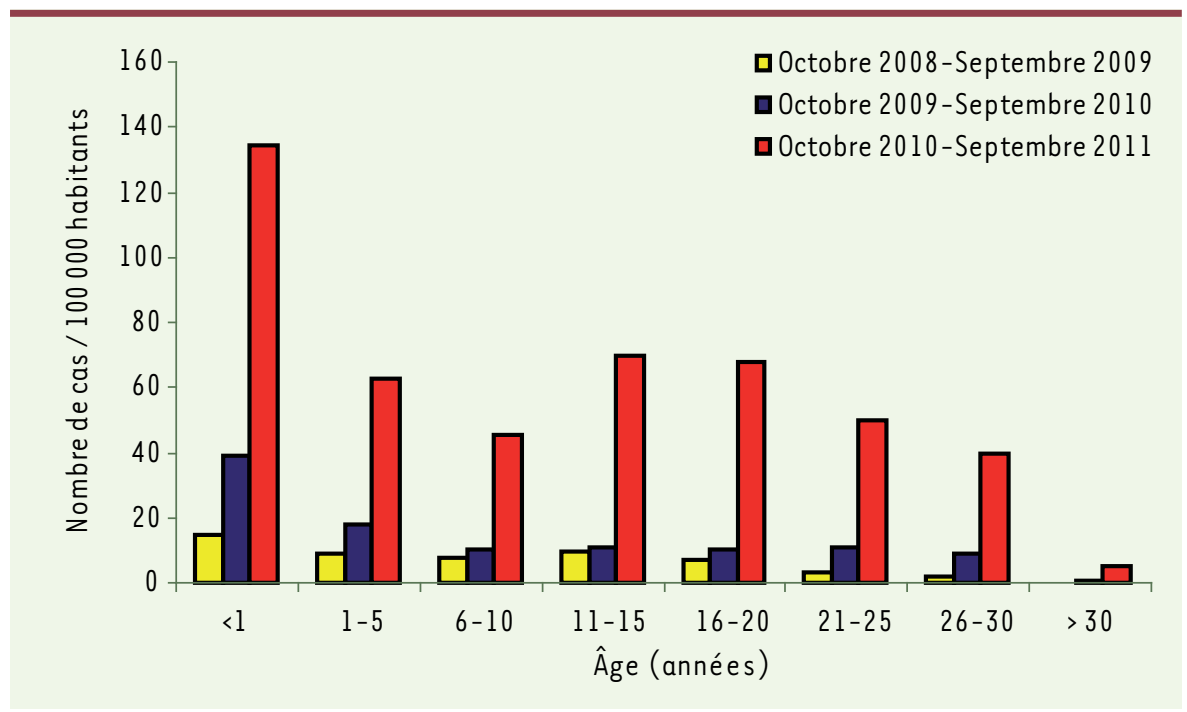

Figure 3. Incidence annuelle de la rougeole selon l'âge. Évolution au cours des trois vagues épidémiques, France, d'octobre 2008 à septembre 2011 (source: chiffres de déclarations obligatoires, InVS).

Les résultats de l'enquête nationale de séroprévalence réalisée par l'InVS en 2009-2010 confirment cette situation en estimant à $8 \%$ le taux de sujets réceptifs à la rougeole parmi les personnes âgées de 6 à 29 ans, soit environ 1,5 millions de personnes réceptives [9].

Plusieurs éléments laissent penser que l'incidence de l'infection, telle

Si la majorité des cas ( $80 \%$ ) est survenue chez des sujets non vaccinés, un nombre élevé de cas est survenu chez des sujets vaccinés avec une seule dose, en particulier parmi les personnes âgées de 20 à 29 ans. Cette situation a conduit le Haut conseil de la santé publique à recommander en 2011 le rattrapage vaccinal avec deux doses de vaccin RRO pour l'ensemble des personnes nées depuis 1980 [4].

Le CNR a montré qu'un variant du génotype D4 (MVs/Montaigu. FRA/43.08) circulait de façon quasi exclusive à côté de rares autres génotypes. Il a retrouvé, essentiellement lors de la dernière vague, un génotype particulier $\mathrm{G} 3$ émergeant également dans plusieurs pays européens sans que l'on sache précisément comment cette souche y a été introduite [6].

\section{Discussion}

La situation décrite en 2006 et 2007 semblait correspondre à une phase de pré-élimination mais il s'agissait en réalité d'une «lune de miel » prérésurgence. Cette recrudescence était prévisible - et l'avait d'ailleurs été par des travaux de modélisation [7] - en raison de la couverture vaccinale (CV) antirougeoleuse insuffisante en France permettant la constitution progressive d'un réservoir important de personnes réceptives au virus dans la population.

Si la couverture vaccinale RRO des nourrissons a augmenté ces dernières années, elle reste encore insuffisante. Elle est à 24 mois de $90 \%$ pour une dose et de $54 \%$ pour deux doses (Échantillon généraliste des bénéficiaires CNAMTS/InVS, enfants nés en 2009, données provisoires au 31/12/2011, à paraître), alors que les objectifs du plan d'élimination sont, à cet âge, des couvertures vaccinales de $95 \%$ et $80 \%$, respectivement, pour la $1^{\text {re }}$ et la $2^{\mathrm{e}}$ dose. II existe, au-delà de deux ans, un rattrapage vaccinal. Toutefois, les enquêtes en milieu scolaire montraient une couverture avec la $2^{\mathrm{e}}$ dose encore insuffisante, de $44 \%$ à 6 ans en 2006 et de $85 \%$ à 11 ans en 2008 (pour la $1^{\text {re }}$ dose, elle est respectivement de $93 \%$ et $96 \%$ ) $[8]^{3}$.

${ }^{3}$ http://www.invs.sante.fr/Dossiers-thematiques/Maladies-infectieuses/Maladies-a-prevention-vaccinale/Couverture-vaccinale/Donnees/Rougeole-rubeole-oreillons qu'elle est estimée à partir des chiffres des déclarations obligatoires, est sous-estimée de plus de $50 \%$ [10]. Cette sous-déclaration a sans doute fluctué dans le temps, probablement différente selon l'âge et plus importante pour les cas vus en médecine libérale que pour ceux vus à l'hôpital. D'autre part, un meilleur taux de déclaration des cas vus à l'hôpital expliquerait aussi probablement en partie les proportions plus élevées de complications graves que nous avons observées par comparaison avec les données de la littérature; la même remarque inverse peut être faite concernant la faible part des complications habituellement vues en ville comme les otites et les diarrhées [11, 12]. À noter que les hospitalisations exposent au risque de transmission nosocomiale comme décrit récemment [13], et ceci doit conduire au renforcement du rattrapage des vaccinations parmi les professionnels de santé selon les recommandations vaccinales actuelles [4].

Plusieurs pays européens ont été touchés par des épidémies de rougeole au cours des dernières années. En 2010, la France était l'un des pays européens avec la plus forte incidence (après la Bulgarie avec plus de 20000 cas) et, en 2011 , le plus affecté puisque près de $80 \%$ des cas déclarés en Europe l'étaient en France $[14,15]$. Des pays limitrophes comme la Suisse ont vu augmenter le nombre de cas de rougeole début 2011 [16]. La France a contribué à l'exportation de la rougeole dans plusieurs pays d'Europe, mais également dans des zones comme celle des Amériques où la rougeole était en voie de certification de son élimination [17]. La surveillance de la rougeole et le contrôle de l'environnment de chaque patient doivent être une priorité dans ces régions, qui incluent les départements français d'Amérique (Martinique, Guadeloupe et Guyane). 


\section{Conclusion}

À la suite de l'observation de premiers cas groupés en milieu scolaire ainsi qu'en milieu de soins au printemps 2008, des actions de sensibilisation au risque de résurgence de la rougeole ont été menées par les autorités de santé et les professionnels de santé publique, conformément au plan d'éradication de cette infection de 2005. Elles n'ont toutefois pas été suffisantes pour freiner la circulation du virus qui a progressivement diffusé en population générale. Compte tenu de la grande contagiosité de la rougeole, seules des interventions vaccinales permettant d'atteindre rapidement un niveau d'immunité d'au moins $95 \%$ chez les populations ou collectivités concernées sont à même de contrôler les épidémies [18]. Depuis fin 2010 et surtout depuis l'automne 2011, les messages d'information ont été renforcés vis-à-vis de la population générale et des acteurs de santé, ciblant également les populations les plus fragiles ainsi que celles de plus grande réceptivité, notamment en termes de tranches d'âge ou de région géographique (http://info-rougeole.fr/).

À partir du mois d'août 2011, on a observé une diminution du nombre de cas déclarés, qui reste stable autour d'une centaine par mois lors de la saison hiverno-printanière 2011-2012, sans recrudescence en mars-avril, contrairement aux années précédentes.

II reste toutefois un réservoir de sujets réceptifs suffisant pour maintenir la transmission du virus, même à bas bruit, et se pose la question de la capacité de la France à atteindre l'objectif d'élimination de la rougeole en 2015. En effet, tant que la couverture du nourrisson n'atteindra pas le niveau requis de $95 \%$ et que le rattrapage des cohortes d'enfants plus âgés et de jeunes adultes ne sera pas renforcé, on risque d'observer une alternance de vagues épidémiques suivies de périodes de «lune de miel ». L'objectif d'augmentation de la couverture vaccinale est, non seulement, de protéger directement les sujets vaccinés, mais également de protéger de la contamination les sujets vulnérables, à risque de formes graves, à travers une immunité de groupe, en particulier les personnes ne pouvant bénéficier de la vaccination (enfants de moins d'un an, immunodéprimés, femmes enceintes). Cette immunité de groupe ne pourra être obtenue que grâce à des niveaux de couverture vaccinale très élevés dans toute la population ciblée par les recommandations actuelles. $\diamond$

\section{SUMMARY}

\section{Measles in France}

From January 2008 to May 2012, over 22,000 cases of measles were reported in France. The highest incidence rate was observed in children below one year of age. Over $50 \%$ of cases were reported in young adults. Almost 5,000 patients were hospitalised including 1,023 with severe pneumonia, 27 with encephalitis and/or myelitis: 10 died. This situation is linked to insufficient and heterogeneous vaccination coverage with pockets of susceptible people allowing virus circulation. Although the vaccine coverage in children has now improved for both doses, the issue of convincing young susceptible adults to catch up for measles vaccination remains critical, if the elimination target is to be met, and in order to protect the most vulnerable population unable to benefit from this vaccination (children below 1 year, immunodeficient people, pregnant women). $\diamond$

\section{LIENS D’INTÉRÊT}

Les auteurs déclarent n'avoir aucun lien d'intérêt concernant les données publiées dans cet article.

\section{POUR EN SAVOIR PLUS}

http://www.invs.sante.fr/Dossiers-thematiques/Maladies-infectieuses/ Maladies-a-declaration-obligatoire/Rougeole

\section{RÉFÉRENCES}

1. Strebel PM, Papania MJ, Dayan GH, Halsey NA. Measles vaccine. In : Plotkin SA, Orenstein WA, Offit PA, eds. Vaccines, $5^{\text {th }}$ ed. St Louis : Saunders, 2008.

2. Wolfson LJ, Grais RF, Luquero FJ, et al. Estimates of measles case fatality ratios: a comprehensive review of community-based studies. Int Epidemiol 2009 ; 38 : 193-205.

3. Ministère de la santé et des solidarités. Plan d'élimination de la rougeole et de la rubéole congénitale en France 2005-2010. Disponible à http:// www.sante.gouv.fr/IMG/pdf/plan_elimination_rougeole.pdf (consulté le 26 mai 2012)

4. Haut conseil de la santé publique. Le calendrier des vaccinations et les recommandations vaccinales 2012 selon l'avis du Haut conseil de la santé publique. Bull Epidemiol Hebd 2012 ; 14-15 : 161-86.

5. Circulaire $n^{\circ}$ DGS/RI1/2009/334 du 4 novembre 2009 relative à la transmission obligatoire de données individuelles à l'autorité sanitaire en cas de rougeole et la mise en œuvre de mesures préventives autour d'un cas ou de cas groupés.

6. Brown KE, Mulders MN, Freymuth F, et al. Appearance of a novel measles G3 strain in multiple european countries within a two month period, 2010. Euro Surveill $2011 ; 16$ : 19852.

7. Lévy-Bruhl D, Maccario D, Richardson S, Guérin N. Modélisation de la rougeole en France et conséquences pour l'âge d'administration de la seconde vaccination rougeole-oreillons-rubéole. Bull Epidemiol Hebd $1997 ; 29: 133-5$.

8. Fonteneau L, Urcun JM, Kerneur C, et al. Couverture vaccinale des enfants âgés de 11 ans scolarisés en CM2, France, 2004-2005. Bull Epidemiol Hebd 2008 ; 51-52: 493-7.

9. Lepoutre A, Antona D, Fonteneau L, et al. Enquête nationale de séroprévalence des maladies infectieuses 2009-2010, lers résultats. $12^{\text {es }}$ Journées nationales d'infectiologie, communication orale, Toulouse, 2011. Med Mal Inf 2011; 41 (suppl 1) : 6

10. Parent du Chatelet I, Antona D, Freymuth F, et al. Spotlight on measles 2010: update on the ongoing measles outbreak in France, 2008-2010. Euro Surveill $2010 ; 15: 19656$.

11. Yasunaga $H$, Shi Y, Takeuchi $M$, et al. Measles-related hospitalizations and complications in Japan, 2007-2008. Intern Med 2010 ; 49: 1965-70.

12. Filia $A$, Brenna A, Pana A, et al. Health burden and economic impact of measles-related hospitalizations in Italy in 2002-2003. BMC Public Health $2007 ; 7: 169$.

13. Carbonne A, Hassani Y, Thiolet JM, et al. Signalements de cas de rougeoles en établissements de santé, reçus au CCLIN Paris-Nord de janvier 2009 à mars 2011. Bull Epidemiol Hebd 2011 ; 34-35 : 385-7.

14. Cottrell S, Roberts RJ. Measles outbreak in Europe. Br Med J 2011 ; $342 \mathrm{~d} 3724$.

15. Steffens I, Martin R, Lopalco P. Spotlight on measles 2010: measles elimination in Europe: a new commitment to meet the goal by 2015. Euro Surveill $2010 ; 15: 19749$.

16. Delaporte $\varepsilon$, Richard JL, Wyler Lazarevic CA, et al. Ongoing measles outbreak, Geneva, Switzerland, January to March 2011. Euro Surveill $2011 ; 16: 19815$.

17. Measles-United States, January-May 20, 2011. Morb Mortal Wkly Rep $2011 ; 60: 666-8$.

18. Nokes DJ, Anderson RM. The use of mathematical models in the epidemiological study of infectious diseases and in the design of mass immunization programmes. Epidemiol Infect 1988 ; 101 : 1-20.

\section{TIRÉS À PART}

D. Antona 